Article Type: Research Papers

\title{
Large-brained birds suffer less oxidative damage
}

CSONGOR. I. VÁGÁSI*†, ORSOLYA VINCZE*†, LAURA PĂTRAȘ

OSVÁTH§, ATTILA MARTON†ףI, LÖRINC BĂRBOS †II, DANIEL SOL**+†, \& PÉTER

L. PAP*†

*MTA-DE “Lendület” Behavioural Ecology Research Group, Department of Evolutionary

Zoology, University of Debrecen, Debrecen, Hungary

$\dagger$ Evolutionary Ecology Group, Hungarian Department of Biology and Ecology, Babeş-Bolyai University, Cluj Napoca, Romania

$\ddagger$ Department of Molecular Biology and Biotechnology, Babeş-Bolyai University, Cluj

Napoca, Romania

§Museum of Zoology, Babeş-Bolyai University, Cluj Napoca, Romania

II 'Milvus Group’ Bird and Nature Protection Association, Tîrgu Mureș, Romania

**CREAF, Sciences Building, Cerdanyola del Vallès, Spain

$\dagger \dagger$ CSIC, Cerdanyola del Vallès, Spain

This article has been accepted for publication and undergone full peer review but has not been through the copyediting, typesetting, pagination and proofreading process, which may lead to differences between this version and the Version of Record. Please cite this article as doi: $10.1111 /$ jeb.12920

This article is protected by copyright. All rights reserved. 
Correspondence: Csongor I. Vágási, MTA-DE “Lendület” Behavioural Ecology Research Group, Department of Evolutionary Zoology, University of Debrecen, Egyetem tér 1, H4032 Debrecen, Hungary.

Tel: +36 52 512-900 / 62331; fax: +36 52 512-941; e-mail: csvagasi@ gmail.com

C. I. VÁGÁSI ET AL. Brain size and oxidative physiology

\section{Abstract}

Large brains (relative to body size) might confer fitness benefits to animals. Although the putative costs of well-developed brains can constrain the majority of species to modest brain sizes, these costs are still poorly understood. Given that the neural tissue is energetically expensive and demands antioxidants, one potential cost of developing and maintaining large brains is increased oxidative stress ('oxidation exposure' hypothesis). Alternatively, because large-brained species exhibit slow-paced life histories, they are expected to invest more into self-maintenance such as an efficacious antioxidative defence machinery ('oxidation avoidance' hypothesis). We predict decreased antioxidant levels and/or increased oxidative damage in large-brained species in case of oxidation exposure, and the contrary in case of oxidation avoidance. We address these contrasting hypotheses for the first time by means of a phylogenetic comparative approach based on an unprecedented dataset of 4 redox state markers from 85 European bird species. Large-brained birds suffered less oxidative damage to lipids (measured as malondialdehyde levels) and exhibited higher total non-enzymatic antioxidant capacity than small-brained birds, while uric acid and glutathione levels were independent of brain size. These results were not altered by potentially confounding variables and did not depend on how relative brain size was quantified. Our findings partially support the 'oxidation avoidance' hypothesis and provide a physiological explanation for the linkage 
of large brains with slow-paced life histories: reduced oxidative stress of large-brained birds can secure brain functionality and healthy lifespan, which are integral to their lifetime fitness and slow-paced life history.

Keywords: antioxidants; brain size; life history; lipid peroxidation; oxidative stress.

\section{Introduction}

A range of fitness benefits have been associated with large brain sizes (relative to body mass) and these served as primary explanation for their evolution. For instance, the 'cognitivebuffer' hypothesis proposes that large brains confer enhanced cognitive skills and flexible behavioural repertoires, which ultimately buffer them against the extrinsic hazards of mortality (e.g. starvation, parasites, predation and social stress; Allman et al., 1993; reviewed by Sol, 2009). Ample comparative evidence demonstrates that big-brained species excel in terms of learning, cognition, innovation and behavioural coping with environmental stressors, and enjoy enhanced survival and longer lifespan in changing or novel environments (e.g. Shultz et al., 2005; Sol et al., 2005, 2007, 2012; González-Lagos et al., 2010; Maklakov et al., 2011; Benson-Amram et al., 2016; reviewed by Sol, 2009). Considering these salutary effects, it is odd that only a small fraction of homeotherm vertebrates possess considerably larger brains than expected from their body mass (Parker, 1990; Isler \& van Schaik, 2009a; b). Costs of a highly-developed central nervous system should be responsible for constraining the evolution of large brains relative to body mass. Yet, except the high energetic needs of brain development and maintenance, there is scant evidence about putative costs, even though these are imperative to understand brain size evolution (Martin, 1981; Parker, 1990; Isler \& van Schaik, 2006b, 2009a; Navarrete et al., 2011).

This article is protected by copyright. All rights reserved. 
The costs of enlarged brains postulated so far are often grounded on the high metabolic expenses of developing and maintaining the neural tissue, which require high rates of oxygen consumption, ion pumping and neurotransmitter synthesis (Parker, 1990; Aiello \& Wheeler, 1995; Isler \& van Schaik, 2006b). This cost can be paid directly by increased metabolic rate (Parker, 1990; Ricklefs, 2004; Isler \& van Schaik, 2006b; but see Isler \& van Schaik, 2006a) and/or increased maternal metabolic turnover (Martin, 1981; Isler \& van Schaik, 2009a; Barton \& Capellini, 2011). It can also be manifested as energetic trade-offs implying that allocation into the brain tissue is saved to the detriment of investment into intestines, other visceral organs, locomotion, adipose tissue or production of soma and progeny (Aiello \& Wheeler, 1995; Isler \& van Schaik, 2006a, 2009a; b; Barton \& Capellini, 2011; Navarrete et al., 2011; Kotrschal et al., 2013).

Oxidative homeostasis, another component of the physiological network, was also found to play a role in brain development and functioning within species (Gilgun-Sherki et al., 2001; Halliwell, 2001; Lin \& Beal, 2006; Dröge \& Schipper, 2007). However, this topic was seldom addressed in evolutionary biology (see Buchanan et al., 2013 and references therein) and thus the understanding of the brain-redox state nexus remained elusive especially in comparisons across several species. Oxidative stress arises when the generation of reactive oxygen species (ROS) by cellular respiration overwhelms the defence capacity of the antioxidative system (reviewed in detail in Monaghan et al., 2009; Pamplona \& Costantini, 2011). Oxidative stress state implies that vital cellular components (proteins, lipids and DNA) can be damaged, and the accumulation of unrepaired oxidative damages was suggested to play at least some role in ageing and a plethora of age-related disorders (around 150) at the organism level in general and nervous system in particular (Finkel \& Holbrook, 2000; Barja, 2004; Halliwell \& Gutteridge, 2007; Buttemer et al., 2010; Clausen et al., 2010; Speakman et al., 2015).

This article is protected by copyright. All rights reserved. 
Here we ask for the first time whether oxidative state measured in the blood tissue is associated with brain size in birds. We propose two alternative hypotheses linking brain size and redox physiology. The 'oxidation exposure' hypothesis argues that an elevated oxidation exposure might arise in large-brained organisms for at least three reasons. First, the high metabolic expenses of large brains might exacerbate the oxidative insults, all else in the redox system being equal (Beckman \& Ames, 1998; see also Burger et al., 2008); though the metabolic theory of oxidative stress and ageing is progressively devalued (Hulbert et al., 2007; Speakman \& Selman, 2011; Speakman et al., 2015). Second, brain development and maintenance demands large amounts of antioxidants (e.g. glutathione; Galván \& Møller, 2011), which might be traded off with the antioxidant protection against oxidation challenges. Third, the neural tissue demands high blood oxygen and glucose levels, which are sources of ROS production (Aiello \& Wheeler, 1995; Gilgun-Sherki et al., 2001; Halliwell, 2001; Galván \& Møller, 2011).

Alternatively, the 'oxidation avoidance' hypothesis postulates that species with large brains should exhibit adaptations that better retard an oxidative stress state to be settled. Better oxidation avoidance can help to shield out potentially long-lasting and adverse carryover effects of oxidative stress. Given that large-brained species feature a slow pace-of-life, and hence rely on a long reproductive life to improve their fitness (Sol et al., 2007, 2012; Sol, 2009; van Schaik et al., 2012), they should gain fitness benefits by investing into selfmaintenance such as oxidative homeostasis (Halliwell \& Gutteridge, 2007; Monaghan et al., 2009). The rationale behind this hypothesis is that oxidative homeostasis is, on one hand, conducive to brain functionality and cognitive resilience, which are integral to the fitness and slow-paced life history of large-brained species (Buchanan et al., 2013), and, on the other hand, might permit longer reproductive lifespan (Finkel \& Holbrook, 2000; Halliwell \& Gutteridge, 2007; Buttemer et al., 2010; Salmon et al., 2010; Speakman et al., 2015). 
We contrasted the 'oxidation exposure' and 'oxidation avoidance' hypotheses by predicting that the former holds if species with large relative brain size feature reduced antioxidant defence and/or enhanced oxidative damage as measured in the blood tissue, while the latter is supported if these relationships are reversed. For this, we conducted a comprehensive phylogenetic comparative analysis based on an unprecedented dataset of four blood redox state markers of 85 European bird species that belong to 37 families and 13 orders (based on taxonomy by Gill \& Donsker, 2016). Three markers describe the nonenzymatic antioxidant defence (total antioxidant status, uric acid and total glutathione), and one marker shows the oxidative damage of lipids (malondialdehyde). Lipid peroxidation was measured for the first time spanning a wide range of wild-living avian taxa, while the antioxidant parameters were the first time assayed for such a large number of European bird species. These markers were chosen because (1) non-enzymatic antioxidants, besides antioxidant enzymes, are deployed to combat free radical insults and might play a role in ageing (Cohen et al., 2007), (2) glutathione is the most significant intracellular, endogenous, non-enzymatic antioxidant with multifaceted physiological effects (Meister \& Anderson, 1983; Galván \& Alonso-Alvarez, 2008) including brain development and maintenance (Gilgun-Sherki et al., 2001; Halliwell, 2001; Galván \& Møller, 2011) and is also integral to the ageing process (Maher, 2005; Rebrin \& Sohal, 2008), and (3) malondialdehyde, which results from the peroxidative degeneration of polyunsaturated fatty acids by ROS, is a widely used marker of oxidative stress (Del Rio et al., 2005; Halliwell \& Gutteridge, 2007; Monaghan et al., 2009), can act as a ROS itself and consequently can trigger oxidative cascades and perpetuate lipid peroxidation (Barja, 2004; Del Rio et al., 2005; Halliwell \& Gutteridge, 2007), and was also linked to ageing (Spiteller, 2007; Pamplona, 2008).

This article is protected by copyright. All rights reserved. 


\section{Materials and methods}

\section{Fieldwork}

We sampled 544 birds belonging to 85 species in Romania between 2011 and 2013 (Table S1). These species represent a diverse set of European birds as they belong to 37 families and 13 orders (based on taxonomy by Gill \& Donsker, 2016). Only a small fraction of species is represented by one sampled individual (11 out of $85,13 \%$; Table S1). The majority of the species were captured at multiple locations (totally 42 in eight counties) and/or during multiple sampling occasions (Table S1). Species were sampled in their breeding phase from late April until early July. The breeding phase was confirmed by females exhibiting clear brood patches. After capture, we banded the birds with metal rings (to exclude repeated sampling of the same individuals) and determined their age (only adults were considered) and sex (except for 28 species that are sexually monomorphic). Then, we took a blood sample (range 30-300 $\mu \mathrm{L}$, depending on body size) by brachial venepuncture into heparinized capillaries. The blood sample drawn from small-sized species usually allowed only one aliquot, therefore different redox state markers were measured from different samples, which makes the sample size to vary per marker per species (Table S1). For the welfare of birds and potential stress-sensitivity of redox markers, bleeding took place as fast as possible after the bird hit the net (bleeding time within $15 \mathrm{~min}$; mean = $9.34 \mathrm{~min}, \mathrm{SD}=4.72$ ). All birds were released in good condition after sampling. The samples were kept in dark cooling boxes at around $4^{\circ} \mathrm{C}$ for less than $10 \mathrm{hs}$ until spun (for 5 mins at 5,000 rpm) to separate the plasma and erythrocyte fractions. The plasma fraction was visually scored for the degree of haemolysis on a 4-point scale (none, weak, intermediate, strong), then plasma was partitioned into aliquots for each marker and all aliquots and the erythrocytes were stored at $-50^{\circ} \mathrm{C}$ until assaying. Laboratory assays (see below) were done following the same protocol and by the same person (LP). The abovementioned standardisation of sample collection and assaying 
minimized the heterogeneity in data due to geographic region, life-history stage, bird's age, stress exposure (samples only from Romania, breeding season and adults, and collected within a short time, respectively) and laboratory protocols. Trapping by mist nets and blood sampling was done as licensed by the Romanian Academy of Sciences (permission no. 2257) and in accordance with current Romanian laws of animal welfare.

\section{Biochemical assays}

We measured three antioxidant markers (total antioxidant status, TAS, uric acid, UA, and total glutathione, tGSH) and a marker of peroxidative damage to lipids (malondialdehyde, MDA, by HPLC). Detailed protocols can be found in the Supporting Information and Bókony et al. (2014). UA significantly positively correlates with TAS (species-level correlation: $r=$ $0.32,95 \%$ CI: $\left.0.12-0.50, t_{82}=3.07, P=0.003\right)$ in agreement with previous studies (Cohen $e t$ al., 2008). To remove the contribution of UA to TAS, we extracted the TAS residuals from an ordinary least squares regression (Cohen et al., 2008) of individual-level measures of TAS on UA and then averaged this per species.

\section{Brain size}

Information on brain mass was obtained from a previously assembled dataset (Sol et al., 2010). Despite concerns raised about combining brain measurements from different sources, this has proved negligible in comparisons across species (Sol et al., 2010). For seven species we had no species-specific brain mass data and thus we instead used genera mean brain mass; however, this did not alter the results (see the Supporting Information). To account for the allometric effects of body mass on brain mass, body mass data were collected from (Dunning, 2008) and the allometric exponent was estimated as the slope of the log brain size on log 
body mass by means of phylogenetic generalized least squares (PGLS) regression. Brain size scales to body mass ${ }^{0.65}(\log$ brain mass $=-2.48+0.65 \times \log$ body mass $)$; the scaling exponent 0.65 being very close to the hypothetic negative allometric exponent 0.67 . Consequently, we derived a mass-corrected brain size dividing original brain mass with body mass ${ }^{0.65}$ (see also Sol et al., 2008). The mass-corrected brain size thus reflects the size of the brain relative to what is expected from body size. The results found by other two alternative measures of relative brain size are coincident and can be found in the Supporting Information.

\section{Confounding variables}

We ruled out the potentially confounding effects of sampling stress (expressed as time elapsed between hitting the net and bleeding), degree of haemolysis, sampling date, body mass, life history and breeding latitude. Details on their relevance and methods are provided in the Supporting Information.

\section{Statistical analyses}

All statistical analyses were carried out in R 3.2 (R Core Team, 2015). To assess whether redox state markers are suitable for multispecies comparison, we tested the species-specificity of these traits by partitioning variance into among- and within-species components using the function 'ICCest' from the R package 'ICC' (Wolak et al., 2011). Since the precision of within-species variance estimation is dependent on sample size, the estimation of intraclass correlation coefficient (ICC; a measure of species-specificity here) in comparative studies with unbalanced sample sizes across species is not equivocal. To cope with this problem, as a first step, species with only one individual sampled per focal redox marker were excluded. Subsequently, for the remaining species, we randomly picked two individuals out of the total 
number of available measurements per species and computed ICC. This routine was iterated 1,000 times, so final ICCs are the average of the 1,000 ICC estimates. Then we restricted ICC calculations to species with at least three, four and five individuals sampled within species. ICC was computed using raw, non-transformed values. We also used an alternative approach building a model for the entire individual-level dataset (i.e. without restriction to any particular sample size within species) with species ID being set as random factor. The variance was partitioned to among- and within-species components ( $V_{\mathrm{a}}$ and $V_{\mathrm{w}}$, respectively) and ICC was computed as $V_{\mathrm{a}} /\left(V_{\mathrm{a}}+V_{\mathrm{w}}\right)$.

To test the 'oxidation exposure' and 'oxidation avoidance' hypotheses, we modelled each of the 4 redox markers as a function of relative brain size and the three potential confounding variables (body mass, developmental time and LCB) as covariates. Additionally, because the redox state markers are expected to covary, those three markers that were not the response in the actual model were also entered as covariates to assess whether they are related to relative brain size independently of each other. Sex was omitted from models since one third of the species could not be sexed in the field. However, the significant speciesspecificity of redox markers (see Results) indicates that conspecifics are more similar to each other than to other species, independent of their sex. All variables were log-transformed to meet the normality assumption, and were scaled to zero mean and unit SD to gain comparable parameter estimates. The full models were reduced to minimal adequate models (MAMs) by backward stepwise elimination with the more permissive criterion of $P<0.1$ in order to retain marginal explanatory terms as well (i.e. $0.05<P<0.1$ ). Although body mass and developmental period were correlated, the fact that their relationship with the response variables were consistent whether the other life-history trait was present or absent in the model suggests that collinearity was not an issue in our models. 
To account for the dependence of species due to shared evolutionary ancestry, we built PGLS models in which phylogenetic signal (Pagel's $\lambda$; Pagel, 1997, 1999) was estimated by maximum likelihood. To control for phylogeny and uncertainties in phylogenetic construction, we retrieved 1,000 phylogenetic trees from birdtree.org (Jetz et al., 2012), with the backbone tree of (Hackett et al., 2008), which were merged into an ultrametric consensus phylogenetic tree using the SumTrees program (Sukumaran \& Holder, 2010). Each PGLS model was based on the entire species pool and we weighted models by within species sample size (i.e. sampling effort) of the focal response variable as implemented in the 'gls' function of the R package 'nlme' (Pinheiro et al., 2015). This was meant to avoid the possibility that well- or poorly-sampled species might alter the results. Furthermore, all analyses were repeated by only using species with at least three individuals sampled within species for the focal response variable, the level at which each redox state marker proved to be species-specific (see Table S2).

Phylogenetic signal of redox parameters was computed using the 'phylosig' function of the R package 'phytools' (Revell, 2012). Strong and weak signal (i.e. $\lambda$ approaches 1 and 0 , respectively) indicates that evolution conforms to or deviates from the Brownian motion model, respectively.

Additional statistical methods can be found in the Supporting Information.

\section{Results}

The redox state markers were found to be species-specific (i.e. have significant ICC values) with both approaches (i.e. based on the entire dataset or with sample size restrictions); though, the ICC of non-enzymatic antioxidant markers were generally low (Table S2). The phylogenetic signal of MDA was marginally (Pagel's $\lambda=0.57, P=0.087$ ) and that of tGSH 
significantly $(\lambda=0.46, P=0.01)$ different from zero, while it did not differ from zero for the other two antioxidant markers (TAS: $\lambda=0.00, P=1.0$; UA: $\lambda=0.24, P=0.37$ ).

The levels of UA and MDA were weakly negatively, while the concentration of tGSH was significantly positively related to body mass. TAS and UA levels increased with higher degree of lipid peroxidative damage (i.e. MDA concentration; Table 1). In addition, residual TAS was also elevated in species with higher MDA level (PGLS, $\beta \pm \mathrm{SE}=0.08 \pm 0.03, t_{84}=$ 2.47, $P=0.016$, Pagel's $\lambda=0.04)$.

Species with large relative brain size featured significantly higher TAS and significantly lower peroxidative damage to lipids (expressed by the concentration of MDA) in concordance with the 'oxidation avoidance' hypothesis (Table 1, Fig. 1; see Table S3 for full models). UA and tGSH, however, were unrelated to relative brain size. These results were robust to the confounding effects of body mass, developmental time, LCB and redox state covariates (Table 1), and held regardless of multiple changes in the modelling procedure (see Sensitivity analyses and Tables S4, S5 and S6 in the Supporting Information).

\section{Discussion}

We found that the four redox state markers are species-specific, i.e. conspecifics resemble each other more (even if they differ in e.g. sampling stress, sampling day or breeding status) than other species, which is a primary requisite of phylogenetic comparisons. We showed that bird species with disproportionally larger brains relative to body mass suffer lower oxidative damage to cell lipids (MDA) and have higher total non-enzymatic antioxidant capacity (TAS) in the peripheral blood during the energetically demanding period of reproduction. However, two individual antioxidant compounds, plasma uric acid (UA) and erythrocyte glutathione (GSH), were not related to brain size. These findings lend partial support for the 'oxidation 
avoidance' hypothesis and refute the hypothesis that large brains bring 'oxidation exposure' costs.

MDA is a reactive carbonyl species, a di-aldehyde intermediate of lipids' peroxidative decomposition. Cardiolipin, the most frequent phospholipid in mitochondrial membranes, has central role in cellular bioenergetics and is highly susceptible to oxidation (Paradies et al., 2011). Therefore, higher lipid peroxidation levels might imply cardiolipin depletion, cellular energetic decay and loss of functionality (Paradies et al., 2011). Furthermore, MDA is a prime promoter of oxidative vicious cycles by perpetuating lipid peroxidation and causing oxidative damages to proteins and DNA as well (Barja, 2004; Halliwell \& Gutteridge, 2007). These oxidative damages are unwanted per se because of their adverse effects on neural and organismal capacity, but also derange other crucial physiological setting points like immune effectors and the insulin/IGF-1 signalling that can also cause further damages for instance in the brain (Dröge \& Schipper, 2007). These multifaceted negative effects of MDA can ultimately jeopardize mitochondrial, cellular and organismal functioning and accelerate ageing (Pamplona, 2008). Species with large relative brain size thus seem to protect better their odds by mitigating such oxidation costs during the oxidatively challenging breeding period.

Studies involving transgenic model organism increasingly suggest that antioxidants might play a minor role in combating oxidative stress and silencing (neural) ageing (e.g. Barja, 2004; Speakman et al., 2015). However, comparative evidence in wild-living birds shows that the level of antioxidants is elevated in species exposed to increased oxidative insults (Cohen et al., 2008). Consistent with this latter study, we found that species with higher levels of MDA also exhibit higher concentrations of TAS in their bloodstream. Therefore, boosted levels of non-enzymatic antioxidants might play a role in mitigating oxidative damage of large-brained species. However, this does not deny that adaptations 
other than non-enzymatic antioxidant defence might also alleviate oxidative stress in largebrained species, including lower ROS generation rate (Pamplona et al., 2005), higher activity of antioxidant enzymes (Clausen et al., 2010), lowered cardiolipin content (Paradies et al., 2011) and lower receptor density for glucocorticoids or IGF-1 (Gilgun-Sherki et al., 2001; Dröge \& Schipper, 2007).

Our results show that the concentration of tGSH is unrelated to brain size, even though a positive association was previously postulated (Galván \& Møller, 2011). GSH is a tri-peptide ubiquitous in virtually all cells, a key intracellular antioxidant per se due to the thiol group and it is also required as substrate by the antioxidant enzyme glutathione peroxidase (Gilgun-Sherki et al., 2001). GSH preserves brain functionality during ageing and protects the blood-brain barrier (Halliwell, 2001) and the level of reduced GSH decreases with ageing in brain and other tissues as well (Dröge \& Schipper, 2007). The downside of GSH-mediated antioxidant potential is its difficulty to bypass the blood-brain barrier (Gilgun-Sherki et al., 2001). Interestingly, vitamin C and E, which are significant components of TAS, can cross the blood-brain barrier, have large and average concentrations in the brain, respectively, and both were found to be important in neural protection against oxidative insults and neurodegenerative diseases, alone or in cocktails (Gilgun-Sherki et al., 2001). These differences among antioxidants might explain the positive association between TAS and brain size and the lack of association between tGSH and brain size.

At least three mutually non-exclusive explanations can be put forward for the negative covariation between brain size and oxidative stress. First, certain components of the total non-enzymatic antioxidants are derived from food. If a large relative brain size enhances the ability to discriminate and choose food resources richer in antioxidants (e.g. via visual acuity; Schaefer et al., 2008 and/or diet generalism; Overington et al., 2011; Ducatez et al., 2015; Sol et al., 2016), this should lead to an association between oxidative damage and relative 
brain size. Second, given that glucocorticoids induce oxidative stress (Costantini et al., 2011), large-brained species might suffer less glucocorticoid-induced oxidative stress if they cope with stress by means of cognitive mechanisms (e.g. anticipation or innovation) rather than by a glucocorticoid response (Lendvai et al., 2013). Finally, the overall energy consumption rates in large-brained animals might be lower than generally assumed: (Pontzer et al., 2014) reported that primates expend on average only $50 \%$ of the energy expected for a mammal of similar mass. This does not contradict that a large brain consumes more energy, as, despite their lower total energy expenditure, primates have basal metabolic rates similar to those of other mammals; however, it does suggest that overall they might suffer less oxidative damage. Whether this is also true in birds requires further analyses (but see Isler \& van Schaik, 2006b).

An important contribution of our findings is that they provide a physiological explanation for the evolutionary linkage between large relative brain size and slow-paced life histories (Sol, 2009; van Schaik et al., 2012) (Fig. 2). In large-brained and long-lived species selection should favour, on one hand, adaptations that ensure a homeostatic development and maintenance of the nervous system and, on the other hand, adaptations that promote longevity (Barja, 2004; van Schaik et al., 2012; Buchanan et al., 2013). Improved oxidation avoidance is a good candidate mechanism to meet both requirements. On one hand, it promotes brain functionality and cognitive resilience, which has high relevance for the fitness of slow-living large-brained species (Buchanan et al., 2013). On the other hand, the ageing process might be at least partly contingent upon the redox state (Finkel \& Holbrook, 2000; Halliwell \& Gutteridge, 2007; Salmon et al., 2010; Galván et al., 2015; Speakman et al., 2015), and the accumulation of ROS-induced oxidative injuries (e.g. mtDNA mutations) in post-mitotic tissues such as the brain are considered relevant causes of ageing in both mammals and birds (Gilgun-Sherki et al., 2001; Barja, 2004; Hyun et al., 2006). This is 
corroborated by our data as we found higher TAS and lower MDA levels in bird species that live longer and feature slow-paced life history (Vágási et al., in prep.). Thus, big-brained birds might benefit not only from reduced extrinsic mortality risk via cognitive buffering, but also from reduced intrinsic hazards via oxidation avoidance. We speculate that this might have implications for the more frequent occurrence of large brains in birds as opposed to mammals (Isler \& van Schaik, 2009b). The peculiarities of the avian redox physiological system confer them two-fold longer lifespan relative to size-matched mammals (Hulbert et al., 2007; Costantini, 2008). Because species with large relative brains have reduced reproductive rates and lifespan partially levels off this loss (Isler \& van Schaik, 2009b), the longer (reproductive) lifespan of birds might contribute to the higher frequency of large brains in birds.

In sum, our study provides support for the hypothesis that large brain size relative to body mass coevolves with an improved resistance to oxidative stress in birds. To assess the generality of our findings, the covariation between brain size and redox state needs to be further validated in other organisms. Taking the outstanding differences in redox physiology and ageing between birds and mammals (Costantini, 2008), but also in brain size (Isler \& van Schaik, 2009b), mammals are an obvious choice for future tests of the hypotheses. It would also be insightful to delve into the proposed mechanisms and to measure redox state markers in post-mitotic tissue as well.

\section{Acknowledgements}

We owe a debt of gratitude to J. Veres-Szászka, K. Sándor, B. Bakó, M. Csiszár and A. Fülöp for fieldwork, M. Banciu and A. Sesarman for biochemical advice and the directorate of the Botanical Garden Cluj for permitting bird capturing. We thank the editor Judith Mank, two 
anonymous reviewers, Neil Metcalfe and Alan A. Cohen for their help to improve the quality of the manuscript. This research was supported by the European Union and the State of Hungary, co-financed by the European Social Fund in the framework of TÁMOP 4.2.4.A/211-1-2012-0001 'National Excellence Program'. The research infrastructure was supported by a CNCSIS research grant (PN II. RU TE 291/2010) of the Romanian Ministry of Education and Research. During writing, CIV was financed by the Postdoctoral Fellowship Programme and the János Bolyai Research Scholarship of the Hungarian Academy of Sciences, PLP by the János Bolyai Research Scholarship of the Hungarian Academy of Sciences and DS by a Proyecto de Investigación CGL2013-47448-P. We declare we have no conflicting interests.

\section{Data accessibility}

Supporting Information Table S1 shows the species, sample sizes per each oxidative stress variable, the number of sampling locations and dates, mean and SD of sampling dates, and body and brain mass data. The species means of the four oxidative state markers are archived at Dryad repository: DOI xyz.

\section{References}

Aiello, L.C. \& Wheeler, P. 1995. The expensive-tissue hypothesis: the brain and the digestive system in human and primate evolution. Curr. Anthropol. 36: 199-221.

Allman, J., McLaughlin, T. \& Hakeem, A. 1993. Brain weight and life-span in primate species. Proc. Natl. Acad. Sci. USA 90: 118-122.

Barja, G. 2004. Free radicals and aging. Trends Neurosci. 27: 595-600.

This article is protected by copyright. All rights reserved. 
Barton, R.A. \& Capellini, I. 2011. Maternal investment, life histories, and the costs of brain growth in mammals. Proc. Natl. Acad. Sci. USA 108: 6169-6174.

Beckman, K.B. \& Ames, B.N. 1998. The free radical theory of aging matures. Physiol. Rev. 78: $547-581$.

Benson-Amram, S., Dantzer, B., Stricker, G., Swanson, E.M. \& Holekamp, K.E. 2016. Brain size predicts problem-solving ability in mammalian carnivores. Proc. Natl. Acad. Sci. USA 113: 2532-2537.

Bókony, V., Lendvai, Á.Z., Vágási, C.I., Pătraș, L., Pap, P.L., Németh, J., et al. 2014. Necessity or capacity? Physiological state predicts problem-solving performance in house sparrows. Behav. Ecol. 25: 124-135.

Buchanan, K.L., Grindstaff, J.L. \& Pravosudov, V. V. 2013. Condition dependence, developmental plasticity, and cognition: implications for ecology and evolution. Trends Ecol. Evol. 28: 290-296.

Burger, J.M.S., Kolss, M., Pont, J. \& Kawecki, T.J. 2008. Learning ability and longevity: a symmetrical evolutionary trade-off in Drosophila. Evolution 62: 1294-1304.

Buttemer, W.A., Abele, D. \& Costantini, D. 2010. From bivalves to birds: oxidative stress and longevity. Funct. Ecol. 24: 971-983.

Clausen, A., Doctrow, S. \& Baudry, M. 2010. Prevention of cognitive deficits and brain oxidative stress with superoxide dismutase/catalase mimetics in aged mice. Neurobiol. Aging 31: 425-433.

Cohen, A., Klasing, K. \& Ricklefs, R. 2007. Measuring circulating antioxidants in wild birds. Comp. Biochem. Physiol. B 147: 110-121.

Cohen, A.A., McGraw, K.J., Wiersma, P., Williams, J.B., Robinson, W.D., Robinson, T.R., et al. 2008. Interspecific associations between circulating antioxidant levels and lifehistory variation in birds. Am. Nat. 172: 178-193.

This article is protected by copyright. All rights reserved. 
Costantini, D. 2008. Oxidative stress in ecology and evolution: lessons from avian studies. Ecol. Lett. 11: 1238-1251.

Costantini, D., Marasco, V. \& Møller, A.P. 2011. A meta-analysis of glucocorticoids as modulators of oxidative stress in vertebrates. J. Comp. Physiol. B 181: 447-456.

Del Rio, D., Stewart, A.J. \& Pellegrini, N. 2005. A review of recent studies on malondialdehyde as toxic molecule and biological marker of oxidative stress. Nutr. Metab. Cardiovasc. Dis. 15: 316-328.

Dröge, W. \& Schipper, H.M. 2007. Oxidative stress and aberrant signaling in aging and cognitive decline. Aging Cell 6: 361-370.

Ducatez, S., Clavel, J. \& Lefebvre, L. 2015. Ecological generalism and behavioural innovation in birds: technical intelligence or the simple incorporation of new foods? $J$. Anim. Ecol. 84: 79-89.

Dunning, J.B.J. 2008. CRC handbook of avian body masses, 2nd ed. CRC Press, Boca Raton, FL.

Finkel, T. \& Holbrook, N.J. 2000. Oxidants, oxidative stress and the biology of ageing. Nature 408: 239-247.

Galván, I. \& Alonso-Alvarez, C. 2008. An intracellular antioxidant determines the expression of a melanin-based signal in a bird. PLoS One 3: e3335.

Galván, I. \& Møller, A.P. 2011. Brain size and the expression of pheomelanin-based colour in birds. J. Evol. Biol. 24: 999-1006.

Galván, I., Naudí, A., Erritzøe, J., Møller, A.P., Barja, G. \& Pamplona, R. 2015. Long lifespans have evolved with long and monounsaturated fatty acids in birds. Evolution 69: $2776-2784$.

Gilgun-Sherki, Y., Melamed, E. \& Offen, D. 2001. Oxidative stress inducedneurodegenerative diseases: the need for antioxidants that penetrate the blood brain 
barrier. Neuropharmacology 40: 959-975.

Gill, F. \& Donsker, D. 2016. IOC World Bird List version 6.1 (F. Gill \& D. Donsker, eds).

González-Lagos, C., Sol, D. \& Reader, S.M. 2010. Large-brained mammals live longer. J. Evol. Biol. 23: 1064-1074.

Hackett, S.J., Kimball, R.T., Reddy, S., Bowie, R.C.K., Braun, E.L., Braun, M.J., et al. 2008. A phylogenomic study of birds reveals their evolutionary history. Science 320: 17631768.

Halliwell, B. 2001. Role of free radicals in the neurodegenerative diseases: therapeutic implications for antioxidant treatment. Drugs Aging 18: 685-716.

Halliwell, B. \& Gutteridge, J.M.C. 2007. Free radicals in biology and medicine, 4th ed. Oxford University Press, Oxford, UK.

Hulbert, A.J., Pamplona, R., Buffenstein, R. \& Buttemer, W.A. 2007. Life and death: metabolic rate, membrane composition, and life span of animals. Physiol. Rev. 87: $1175-1213$.

Hyun, D.-H., Emerson, S.S., Jo, D.-G., Mattson, M.P. \& de Cabo, R. 2006. Calorie restriction up-regulates the plasma membrane redox system in brain cells and suppresses oxidative stress during aging. Proc. Natl. Acad. Sci. USA 103: 19908-19912.

Isler, K. \& van Schaik, C. 2006a. Costs of encephalization: the energy trade-off hypothesis tested on birds. J. Hum. Evol. 51: 228-243.

Isler, K. \& van Schaik, C.P. 2006b. Metabolic costs of brain size evolution. Biol. Lett. 2: $557-560$.

Isler, K. \& van Schaik, C.P. 2009a. The expensive brain: a framework for explaining evolutionary changes in brain size. J. Hum. Evol. 57: 392-400.

Isler, K. \& van Schaik, C.P. 2009b. Why are there so few smart mammals (but so many smart birds)? Biol. Lett. 5: 125-129.

This article is protected by copyright. All rights reserved. 
Jetz, W., Thomas, G.H., Joy, J.B., Hartmann, K. \& Mooers, A.O. 2012. The global diversity of birds in space and time. Nature 491: 444-448.

Kotrschal, A., Rogell, B., Bundsen, A., Svensson, B., Zajitschek, S., Brännström, I., et al. 2013. Artificial selection on relative brain size in the guppy reveals costs and benefits of evolving a larger brain. Curr. Biol. 23: 168-171.

Lendvai, Á.Z., Bókony, V., Angelier, F., Chastel, O. \& Sol, D. 2013. Do smart birds stress less? An interspecific relationship between brain size and corticosterone levels. Proc. $R$. Soc. London B 280: 20131734.

Lin, M.T. \& Beal, M.F. 2006. Mitochondrial dysfunction and oxidative stress in neurodegenerative diseases. Nature 443: 787-795.

Maher, P. 2005. The effects of stress and aging on glutathione metabolism. Ageing Res. Rev. 4: 288-314.

Maklakov, A.A., Immler, S., Gonzalez-Voyer, A., Rönn, J. \& Kolm, N. 2011. Brains and the city: big-brained passerine birds succeed in urban environments. Biol. Lett. 7: 730-732.

Martin, R.D. 1981. Relative brain size and basal metabolic rate in terrestrial vertebrates. Nature 293: 57-60.

Meister, A. \& Anderson, M.E. 1983. Glutathione. Annu. Rev. Biochem. 52: 711-760.

Monaghan, P., Metcalfe, N.B. \& Torres, R. 2009. Oxidative stress as a mediator of life history trade-offs: mechanisms, measurements and interpretation. Ecol. Lett. 12: 75-92.

Navarrete, A., van Schaik, C.P. \& Isler, K. 2011. Energetics and the evolution of human brain size. Nature 480: 91-93.

Overington, S.E., Griffin, A.S., Sol, D. \& Lefebvre, L. 2011. Are innovative species ecological generalists? A test in North American birds. Behav. Ecol. 22: 1286-1293.

Pagel, M. 1997. Inferring evolutionary processes from phylogenies. Zool. Scr. 26: 331-348. Pagel, M. 1999. The maximum likelihood approach to reconstructing ancestral character 
states of discrete characters on phylogenies. Syst. Biol. 48: 612-622.

Pamplona, R. 2008. Membrane phospholipids, lipoxidative damage and molecular integrity: a causal role in aging and longevity. Biochim. Biophys. Acta - Bioenerg. 1777: 12491262.

Pamplona, R. \& Costantini, D. 2011. Molecular and structural antioxidant defenses against oxidative stress in animals. Am. J. Physiol. Regul. Integr. Comp. Physiol. 301: R843R863.

Pamplona, R., Portero-Otín, M., Sanz, A., Ayala, V., Vasileva, E. \& Barja, G. 2005. Protein and lipid oxidative damage and complex I content are lower in the brain of budgerigar and canaries than in mice. Relation to aging rate. Age 27: 267-280.

Paradies, G., Petrosillo, G., Paradies, V. \& Ruggiero, F.M. 2011. Mitochondrial dysfunction in brain aging: role of oxidative stress and cardiolipin. Neurochem. Int. 58: 447-457.

Parker, S.T. 1990. Why big brains are so rare: energy costs of intelligence and brain size in anthropoid primates. In: Language and intelligence in monkeys and apes (S. T. Parker \& K. R. Gibson, eds), pp. 129-154. Cambridge University Press, New York, NY.

Pinheiro, J., Bates, D., DebRoy, S., Sarkar, D. \& R Core Team. 2015. nlme: Linear and Nonlinear Mixed Effects Models. R package version 3.1-120. http://CRAN.Rproject.org/package $=$ nlme.

Pontzer, H., Raichlen, D.A., Gordon, A.D., Schroepfer-Walker, K.K., Hare, B., O’Neill, M.C., et al. 2014. Primate energy expenditure and life history. Proc. Natl. Acad. Sci. USA 111: 1433-1437.

R Core Team. 2015. $R$ : a language and environment for statistical computing. R Foundation for Statistical Computing, Vienna, Austria. http://www.R-project.org/.

Rebrin, I. \& Sohal, R.S. 2008. Pro-oxidant shift in glutathione redox state during aging. Adv. Drug Deliv. Rev. 60: 1545-1552. 
Revell, L.J. 2012. phytools: an R package for phylogenetic comparative biology (and other things). Methods Ecol. Evol. 3: 217-223.

Ricklefs, R.E. 2004. The cognitive face of avian life histories. Wilson Bull. 116: 119-133.

Salmon, A.B., Richardson, A. \& Pérez, V.I. 2010. Update on the oxidative stress theory of aging: does oxidative stress play a role in aging or healthy aging? Free Radic. Biol. Med. 48: $642-655$.

Schaefer, H.M., McGraw, K. \& Catoni, C. 2008. Birds use fruit colour as honest signal of dietary antioxidant rewards. Funct. Ecol. 22: 303-310.

Shultz, S., Bradbury, R.B., Evans, K.L., Gregory, R.D. \& Blackburn, T.M. 2005. Brain size and resource specialization predict long-term population trends in British birds. Proc. $R$. Soc. London B 272: 2305-2311.

Sol, D. 2009. The cognitive-buffer hypothesis for the evolution of large brains. In: Cognitive Ecology II (R. Dukas \& J. M. Ratcliffe, eds), pp. 111-134. University of Chicago Press, Chicago, IL.

Sol, D., Bacher, S., Reader, S.M. \& Lefebvre, L. 2008. Brain size predicts the success of mammal species introduced into novel environments. Am. Nat. 172: S63-S71.

Sol, D., Duncan, R.P., Blackburn, T.M., Cassey, P. \& Lefebvre, L. 2005. Big brains, enhanced cognition, and response of birds to novel environments. Proc. Natl. Acad. Sci. USA 102: 5460-5465.

Sol, D., Garcia, N., Iwaniuk, A., Davis, K., Meade, A., Boyle, W.A., et al. 2010. Evolutionary divergence in brain size between migratory and resident birds. PLoS One 5: e9617.

Sol, D., Maspons, J., Vall-1losera, M., Bartomeus, I., García-Peña, G.E., Piñol, J., et al. 2012. Unraveling the life history of successful invaders. Science 337: 580-583.

Sol, D., Sayol, F., Ducatez, S. \& Lefebvre, L. 2016. The life-history basis of behavioural 
innovations. Philos. Trans. R. Soc. London B 371: 20150187.

Sol, D., Székely, T., Liker, A. \& Lefebvre, L. 2007. Big-brained birds survive better in nature. Proc. R. Soc. London B 274: 763-769.

Speakman, J.R., Blount, J.D., Bronikowski, A.M., Buffenstein, R., Isaksson, C., Kirkwood, T.B.L., et al. 2015. Oxidative stress and life histories: unresolved issues and current needs. Ecol. Evol. 5: 5745-5757.

Speakman, J.R. \& Selman, C. 2011. The free-radical damage theory: Accumulating evidence against a simple link of oxidative stress to ageing and lifespan. BioEssays 33: 255-259.

Spiteller, G. 2007. The important role of lipid peroxidation processes in aging and age dependent diseases. Mol. Biotechnol. 37: 5-12.

Sukumaran, J. \& Holder, M.T. 2010. DendroPy: A Python library for phylogenetic computing. Bioinformatics 26: 1569-1571.

van Schaik, C.P., Isler, K. \& Burkart, J.M. 2012. Explaining brain size variation: from social to cultural brain. Trends Cogn. Sci. 16: 277-284.

Wolak, M.E., Fairbairn, D.J. \& Paulsen, Y.R. 2011. Guidelines for estimating repeatability. Methods Ecol. Evol. 3: 129-137.

This article is protected by copyright. All rights reserved. 


\section{Tables and figure captions}

Table 1 Minimal adequate PGLS models about the relationship between redox state markers (i.e. antioxidants and oxidative damage) and brain size. Pagel's $\lambda$ of each model is provided in brackets beside the response variables. $N=84$ species for each model. Significant effects are marked in bold.

\begin{tabular}{llllc}
\hline Response & Predictor & $\beta \pm$ SE & $t$ & $P$ \\
\hline TAS $(\lambda=0.00)$ & Intercept & $1.04 \pm 0.17$ & 5.98 & $<0.001$ \\
& LCB & $0.09 \pm 0.05$ & 1.81 & 0.074 \\
& Relative brain size & $0.12 \pm 0.05$ & 2.42 & $\mathbf{0 . 0 1 8}$ \\
& MDA & $0.26 \pm 0.03$ & 7.59 & $<\mathbf{0 . 0 0 1}$ \\
UA $(\lambda=0.32)$ & Intercept & $5.54 \pm 2.01$ & 2.75 & 0.007 \\
& Body mass & $-1.16 \pm 0.59$ & 1.97 & 0.053 \\
& MDA & $3.04 \pm 0.36$ & 8.40 & $<\mathbf{0 . 0 0 1}$ \\
tGSH $(\lambda=0.28)$ & Intercept & $10.61 \pm 1.05$ & 10.08 & $<0.001$ \\
& Body mass & $3.11 \pm 0.72$ & 4.34 & $<\mathbf{0 . 0 0 1}$ \\
& Intercept & $1.00 \pm 0.44$ & 2.24 & 0.028 \\
& Body mass & $-0.25 \pm 0.13$ & 1.88 & 0.064 \\
& Relative brain size & $-0.32 \pm 0.12$ & 2.75 & $\mathbf{0 . 0 0 7}$ \\
& TAS & $0.83 \pm 0.18$ & 4.66 & $<\mathbf{0 . 0 0 1}$ \\
& UA & $0.10 \pm 0.02$ & 5.74 & $<\mathbf{0 . 0 0 1}$ \\
& & & & \\
\hline
\end{tabular}

This article is protected by copyright. All rights reserved. 
Fig. 1 The relationship between relative brain size and (a) total antioxidant status and (b) oxidative damage (MDA). The slopes are extracted from MAMs presented in Table 1. Dot sizes are proportional to within species sample size of oxidative markers plotted without bearing on the fit.

Fig. 2 The brain size-oxidative state-life history triangle. The association of large relative brain size with slow life-history pace might be underpinned by the oxidative stress being lower in large-brained species and higher in species with fast life-history pace.

This article is protected by copyright. All rights reserved. 
Fig. 1
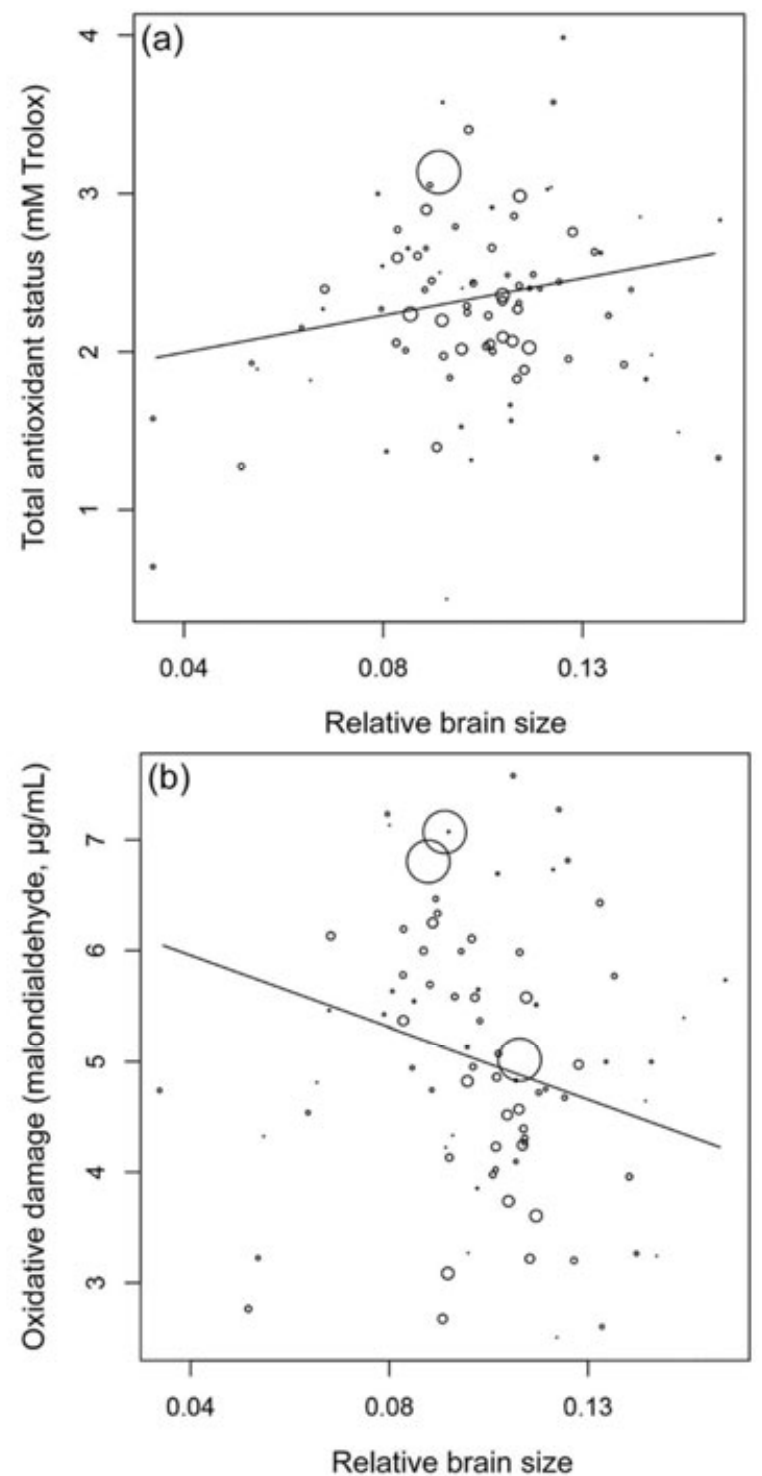

Fig. 2

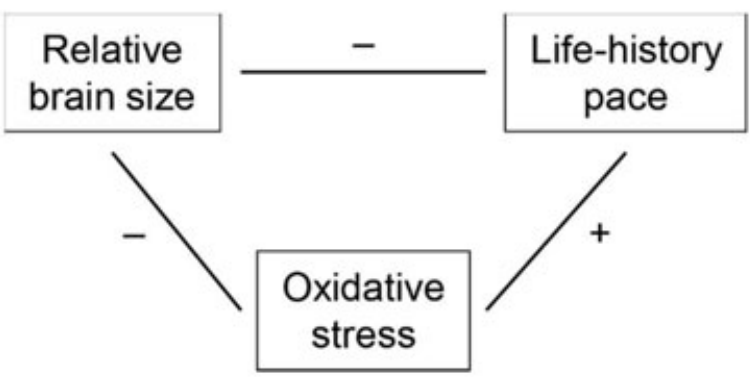

This article is protected by copyright. All rights reserved. 